\title{
How has the genomics era impacted schistosomiasis drug discovery?
}

\begin{abstract}
"A community effort will almost certainly be necessary for the identification of new lead compounds and therefore information and reagent sharing will be important to ensure that the goals of the schistosomiasis drug research community are achieved."
\end{abstract}

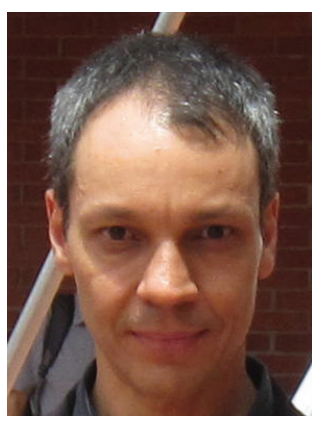

Guilherme Oliveira Author for correspondence: Genomics \& Computational Biology Group, René Rachou Research Center, Oswaldo Cruz Foundation, Av. Augusto de Lima 1715, Belo Horizonte, MG 30190-002, Brazil oliveira@cpqrr.fiocruz.br

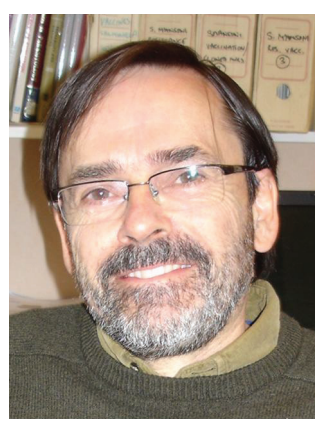

Raymond J Pierce Center for Infection \& Immunity of Lille (CIIL), INSERM U1019-CNRS UMR 8204, Université de Lille, Institut Pasteur de Lille, F-59000 Lille, France sure. Since PZQ is the only drug available, the emergence of drug resistance is extremely concerning [7].

The development of new control tools to combat schistosomiasis have for a long time relied on empirical approaches based on little or no knowledge of the targets, and gene-by-gene methods to further the molecular understanding of schistosome information of interest is being generated by several groups concerning the genetic variability of specimens from endemic sites and the genetics of drug resistance. It has been observed that under praziquantel pressure there is decrease in the genetic diversity of a laboratory schistosome population [8]. With the use of linkage mapping the gene coding for sulfotranferase was described as being responsible for resistance to oxamniquine [9]. Recently, the ability to conduct deep exome sequencing and SNP mapping at high resolution has provided a new tool

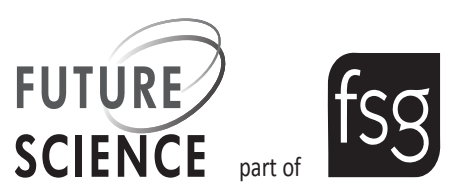


for the identification of genes involved with drug resistance [10]. We anticipate that, in the near future, the genes involved with phenotypes of interest will be identified using these methodologies.

Efforts to explore current genome data have included the use of computational tools for the discovery of novel drug targets and the development of new antischistosomal agents. This has been demonstrated by the Open Source Drug Discovery Consortium, which has identified compounds with activity toward a putative known target, TGR, using machine learning tools and cheminformatics [11].

Computational approaches to neglected disease drug discovery have been enhanced by the availability of resources such as the open access database TDRTargets. By combining predicted protein datasets from genome data with chemical entities derived from public sources, TDRTargets provides researchers with the tools needed to combine queries for protein targets and bioactive compounds. This resource is largely designed to enable drug repositioning and repurposing across different parasite species. For example, using the tools available in TDRTargets, one could query for targets in one organism that shows essentiality in a different species and for which there are known inhibitors, in addition to adding druggability and assayability indices and limiting the results to manually curated compounds [12].

\section{"Overall, the use of public resources, such as those identified above, have enabled researchers to identify and prioritize drug targets..."}

Researchers have also used metabolic modeling software, such as Pathway Tools, to study the metabolic pathways in Schistosoma mansoni and in turn uncover new drug targets. Pathway Tools is capable of predicting an organism's metabolic pathways from its genome by comparison to MetaCyc, a reference pathways database. In a study that was the first to describe the S. mansoni genome, Pathway Tools was used to identify critical points, termed chokepoints, which were demonstrated to be druggable targets [13].

Overall, the use of public resources, such as those identified above, have enabled researchers to identify and prioritize drug targets and, with the huge amounts of genomic data being generated, it is clear that these resources will be essential in helping to accelerate neglected disease drug discovery.

Driving these efforts toward, at the very least, obtaining lead compounds is a challenge, especially for individual research groups. In recent years, strategies to enhance the drug pipeline have been put into place with the establishment of large consortia, such as A-ParaDDisE [14,15]. Such consortia rely on pooling expertise from a range of disciplines in order to work more effectively toward a common goal. These consortia often involve collaboration with industry, which is arguably essential for creating a successful drug pipeline that will deliver a sufficiently large number of compounds to be tested and decrease the cost of preclinical testing. A-ParaDDisE researchers have drawn on the knowledge gained through the development of HMEs as targets in cancer in order to establish these enzymes as targets in schistosomiasis, malaria, leishmaniasis and Chagas disease. HMEs perform covalent modifications of amino acid residues on the N-terminal tails of histones by adding or removing marks, such as methyl or acetyl groups. Histones in turn regulate gene activity by changing chromatin architecture. It has been shown, for example, that deacetylation of histones by HDACs results in tighter wrapping of the DNA, which in turn is associated with the global downregulation of gene transcription. HDACs, in addition to acting on histones, also deacetylate other cytoplasmic proteins. Such activities may be essential in the regulation of the complex schistosome life cycle and enzymes involved in these processes have been shown to be crucial for the growth and differentiation of the parasite [16]. The central role of one HDAC, SmHDAC8, was validated using a phenotypic screen by RNAi to knock down gene expression. An active enzyme was produced and structure data derived from a protein crystal, with or without generic inhibitors. With such data, additional new inhibitor structures were proposed using in silico tools and new small molecules were produced that were found to be active on live worms in culture [16]. This approach is being pursued, in additional to high-throughput screening, to target other HMEs in schistosomes, such as Sirtuins, and for HMEs of other parasites [17,18].

Target identification and establishing drug candidates in the drug discovery process is a difficult task; however, seeing a candidate drug through the safety and drug metabolism phase, and later to clinical trials is an even greater one. With this in mind, the challenge that we are tasked with is the identification of promising candidate drugs that can successfully navigate the 'obstacle course' of toxicology and bioavailability testing and clinical trials and that are inexpensive enough to permit their use in endemic countries.

This special focus issue provides in-depth discussion of the various efforts being undertaken toward populating the schistosomiasis drug development pipeline. A community effort will almost certainly be necessary for the identification of new lead compounds and therefore information and reagent sharing will be important to ensure that the goals of the schistosomiasis drug research community are achieved. 


\section{Financial \& competing interests disclosure}

The authors have no relevant affiliations or financial involvement with any organization or entity with a financial interest in or financial conflict with the subject matter or materials discussed in the manuscript. This includes employment,

\section{References}

1 King CH, Dickman K, Tisch DJ. Reassessment of the cost of chronic helmintic infection: a meta-analysis of disabilityrelated outcomes in endemic schistosomiasis. Lancet 365(9470), 1561-1569 (2005).

2 Gouveia MJ, Santos J, Brindley PJ et al. Estrogen-like metabolites and DNA-adducts in urogenital schistosomiasisassociated bladder cancer. Cancer Lett. 359(2), S0304-S3835 (2015).

3 Gryseels B, Polman K, Clerinx J, Kestens L. Human schistosomiasis. Lancet 368(9541), 1106-1118 (2006).

4 King CH. Parasites and poverty: the case of schistosomiasis. Acta Trop. 113(2), 95-104 (2010).

5 Redman CA, Robertson A, Fallon PG et al. Praziquantel: an urgent and exciting challenge. Parasitol. Today 12(1), 14-20 (1996).

6 Sabah AA, Fletcher C, Webbe G, Doenhoff MJ. Schistosoma mansoni: chemotherapy of infections of different ages. Exp. Parasitol. 61(3), 294-303 (1986).

7 Bennett JL, Day T, Liang FT, Ismail M, Farghaly A. The development of resistance to anthelmintics: a perspective with an emphasis on the antischistosomal drug praziquantel. Exp. Parasitol. 87(3), 260-267 (1997).

8 Coeli R, Baba EH, Araujo N, Coelho PM, Oliveira G. Praziquantel treatment decreases Schistosoma mansoni genetic diversity in experimental infections. PLoS Negl. Trop. Dis. 7(12), e2596 (2013).

9 Valentim CL, Cioli D, Chevalier FD et al. Genetic and molecular basis of drug resistance and species-specific drug action in schistosome parasites. Science 342(6164), 1385-1389 (2013). consultancies, honoraria, stock ownership or options, expert testimony, grants or patents received or pending, or royalties.

No writing assistance was utilized in the production of this manuscript.

10 Chevalier FD, Valentim CL, LoVerde PT, Anderson TJ. Efficient linkage mapping using exome capture and extreme QTL in schistosome parasites. BMC Genomics 15, 617 (2014).

11 Gaba S, Jamal S, Scaria V. Open Source Drug Discovery Consortium. Cheminformatics models for inhibitors of Schistosoma mansoni thioredoxin glutathione reductase. Scientific World J. 2014, 957107 (2014).

12 Crowther GJ, Shanmugam D, Carmona SJ et al. Identification of attractive drug targets in neglected-disease pathogens using an in silico approach. PLoS Negl. Trop. Dis. 4(8), e804 (2010).

13 Berriman M, Haas BJ, LoVerde PT et al. The genome of the blood fluke Schistosoma mansoni. Nature 460 (7253), 352-358 (2009).

14 Pierce RJ, Dubois-Abdesselem F, Lancelot J, Andrade L, Oliveira G. Targeting schistosome histone modifying enzymes for drug development. Curr. Pharm. Des. 18(24), 3567-3578 (2012).

15 Anti-parasitic drug discovery in epigenetics. http://aparaddise.cebio.org/

16 Marek M, Kannan S, Hauser AT et al. Structural basis for the inhibition of histone deacetylase 8 (HDAC8), a key epigenetic player in the blood fluke Schistosoma mansoni. PLoS Pathog. 9(9), e1003645 (2013).

17 Lancelot J, Caby S, Dubois-Abdesselem F et al. Schistosoma mansoni Sirtuins: characterization and potential as chemotherapeutic targets. PLoS Negl. Trop Dis. 7(9), e2428 (2013).

18 Cabezas-Cruz A, Lancelot J, Caby S, Oliveira G, Pierce RJ. Epigenetic control of gene function in schistosomes: a source of therapeutic targets? Front Genet. 5, 317 (2014). 